\title{
LA GRAN MODERACIÓN Y EL RIESGO INFLACIONARIO UNA MIRADA DESDE LAS ECONOMÍAS EMERGENTES*
}

\author{
José De Gregorio
}

Hay un vasto debate sobre las causas de la "gran moderación”, fenómeno observado a partir de mediados de los ochenta en las economías industriales y que ha significado una reducción de la volatilidad no sólo de la inflación sino también del producto. Las explicaciones van desde la buena suerte hasta las buenas políticas. En este documento se argumenta que la política monetaria fue un determinante importante de este fenómeno. La diferencia en tiempo en que ocurre la estabilización entre países desarrollados y emergentes y la coincidencia con la conquista de la inflación, apoyan la idea de una dirección causal desde el control inflacionario hacia una menor volatilidad de la actividad y los precios. Así, las mejoras en el manejo monetario contribuyen tanto a la estabilidad de los precios como del ciclo económico. A su vez, se trata el tema de la mantención de la estabilidad en un contexto de severos riesgos inflacionarios, producto de uno de los más grandes shocks inflacionarios en el período post segunda guerra mundial. Lo más probable, se señala, es que el shock de precios persista por un buen tiempo y que algunos efectos de

JoSÉ DE GRegorio. Presidente del Banco Central de Chile.

* Agradezco los aportes y sugerencias de Christopher Neilson y Andrea Tokman.

Discurso de apertura de la X Conferencia Económica Mundial realizada en Ginebra el 6 de mayo de 2008.

Estudios Públicos, 110 (otoño 2008). 
segunda vuelta resulten inevitables. Se sostiene que la política monetaria se debe preocupar — y ocupar — de la dinámica de la inestabilidad, la que puede generar aumentos de la inflación que luego serán demasiado costosos de contener. Asimismo, se hace hincapié en la necesidad de asegurar que los beneficios logrados durante la fase de la gran moderación se conserven, pues sería una irresponsabilidad retornar a los tiempos de inestabilidad en aras de ganancias de corta duración.

$\mathrm{E}$ un honor dirigirme a ustedes en este importante encuentro. En lo personal, esta es una reunión especial, pues contribuí, junto a Barry Eichengreen, Takatoshi Ito y Charles Wyplosz, a escribir los primeros reportes de esta serie de conferencias en 1999. Fue entretenido y un desafío intelectual trabajar con semejante equipo de colegas y en un tema tan relevante.

En aquel primer Informe Ginebra hablamos de la necesidad de reformar el FMI haciéndolo más autónomo, rasgo que valoramos en los bancos centrales, y responsable en la rendición de cuentas, elemento esencial para que la autonomía sea efectiva. En el futuro me gustaría elaborar más estos temas, pero hoy me centraré en un debate más urgente en esta coyuntura macroeconómica: la política monetaria, la estabilidad y los riesgos actuales de un desborde inflacionario a nivel mundial, precisamente el tema de esta Conferencia.

Comenzaré refiriéndome a la Gran Moderación, expresión que describe la disminución de la volatilidad del producto y de la inflación en las economías industrializadas en los últimos veinte años. Numerosos estudios han identificado los factores que están tras este fenómeno, uno de los cuales es la política monetaria. Yo argumentaré que éste es el factor fundamental, y no sólo uno de muchos factores potencialmente importantes. Mi hipótesis se basa en que en los países en desarrollo la Gran Moderación ocurrió hace unos diez años, y coincidió con el logro de la inflación baja. La secuencia en que ocurrieron los eventos apoya la hipótesis de causalidad de control de la inflación a reducción de volatilidad inflacionaria y de producto. Así, el perfeccionamiento de la política monetaria ayuda a lograr la estabilidad no sólo de los precios sino también del producto, es decir, contribuye a suavizar los ciclos económicos. Mi opinión es que el largo tiempo transcurrido entre la Gran Moderación de los países industrializados y la Gran Moderación de los países en desarrollo levantó dudas sobre la validez y generalidad de las hipótesis que la atribuían a la buena suerte o a la 
existencia de un shock positivo común a todos, como la caída del precio del petróleo o el desarrollo de tecnologías como el manejo automatizado de inventarios.

El segundo tema que abordaré es la perspectiva de mantener la estabilidad en el contexto de riesgo inflacionario severo. De hecho, en los últimos trimestres hemos sido testigos de uno de los shocks inflacionarios más violentos desde la Segunda Guerra Mundial. Es la amenaza más seria a la estabilidad del ciclo económico y a la inflación baja y estable que hemos visto. Hasta hoy, el shock ha tenido un impacto enorme en los más pobres, por la drástica alza de los alimentos esenciales. Más aún, si la inflación continúa, el costo en términos del ciclo económico puede volverse intolerable. Concluiré con algunas reflexiones sobre los desafíos con que tendrá que lidiar la política monetaria.

\section{La Gran Moderación}

La drástica disminución de la volatilidad macroeconómica que hemos visto en Estados Unidos y alrededor del mundo en las últimas décadas ha sido profusamente documentada por estudios empíricos. Kim y Nelson (1999) y Blanchard y Simon (2001) estuvieron entre los primeros en llamar la atención hacia este fenómeno que luego se conoció como la Gran Moderación. Blanchard y Simon encontraron, para Estados Unidos, que la variabilidad del crecimiento trimestral del producto real había caído a la mitad desde mediados de los ochenta, y que la variabilidad de la inflación se había reducido en dos tercios. Tanto la volatilidad del producto como la volatilidad de la inflación experimentaron caídas similares en la mayoría de los países del G-7, y más o menos al mismo tiempo (Stock y Watson, 2003).

Aunque sabemos que Estados Unidos logró controlar la inflación adoptando medidas de política monetaria, todavía se debate qué ocasionó la disminución persistente de la volatilidad tanto del producto como de la inflación. A grandes rasgos, las distintas razones se pueden clasificar en tres grupos:

- El primer argumento es que la Gran Moderación se produjo porque los shocks que golpearon la economía fueron más suaves y menos frecuentes. Esto implicaría que la reducción observada en la volatilidad macroeconómica se debería a la buena suerte y duraría hasta que aparecieran shocks más graves o cambiara la suerte. Muchos estudios han respaldado esta hipótesis de la buena suerte (Ahmed et al., 2002). 
- Una segunda explicación —relacionada con la anterior- se centra en los cambios estructurales y tecnológicos que son de carácter permanente, pero que no fueron la causa directa ni fueron controlados por las políticas macroeconómicas. Por ejemplo, un avance tecnológico que a menudo se menciona en los debates sobre la volatilidad del producto es el mejor manejo de las existencias. Si una parte significativa de la dinámica del ciclo económico obedece a las fluctuaciones de los inventarios, las tecnologías de respuesta "justo a tiempo" y el mejor manejo de las existencias en general reduciría la volatilidad del ciclo (McConnell y Pérez-Quiroz, 2000; Cecchetti et al., 2005). Otro importante cambio estructural tiene que ver con la globalización e integración económica mundial, como se aprecia en el Informe Ginebra elaborado para esta Conferencia (Gerlach et al., 2008). Por ejemplo, Cavallo (2007) subraya que la apertura comercial ha reducido la volatilidad del producto y, en consecuencia, las economías que son más abiertas son a la vez más estables. Esta evidencia refuta la noción antigua de que las economías más abiertas están más expuestas a la volatilidad. El argumento de Cavallo es que, al ser más abierta, una economía tiene más oportunidades de ajustarse a los shocks internacionales. En la línea de este argumento, el notable incremento de la apertura que ha ocurrido en el mundo debería traducirse en mayor estabilidad para todos los países ${ }^{1}$.

- Un tercer y último factor que quisiera subrayar tiene relación con una mejor política monetaria. Estas reformas han conseguido reducir no sólo el nivel sino también la volatilidad de la inflación. La Figura № 1 muestra promedios de crecimiento e inflación para un amplio conjunto de países en desarrollo ${ }^{2}$, y la Figura $\mathrm{N}^{0} 2$ describe la volatilidad del producto y la inflación. Vemos que la volatilidad descendió en las economías en desarrollo recién a mediados de los noventa, casi una década después de la Gran Moderación de las desarrolladas. Podemos decir, entonces, que según esta evidencia hay un descalce en el tiempo entre la gran moderación de los países en vías de desarrollo y los desarrollados.

La hipótesis de la "buena suerte" y los factores estructurales no son una buena explicación para este fenómeno. Si la volatilidad hubiera disminuido únicamente por buena fortuna, entonces sólo los países desarrolla-

\footnotetext{
${ }^{1}$ Kent et al. (2005) analizan también el rol de las reformas a los mercados de productos y de trabajo en la volatilidad del producto para una muestra de veinte países miembros de la OCDE. Para evidencia sobre Chile y estimaciones de VAR de forma reducida, véase Betancour et al. (2008).

2 Se trata de setenta y tres países clasificados por el IFS del FMI como "economías en desarrollo” y para los cuales se tiene información desde 1975.
} 


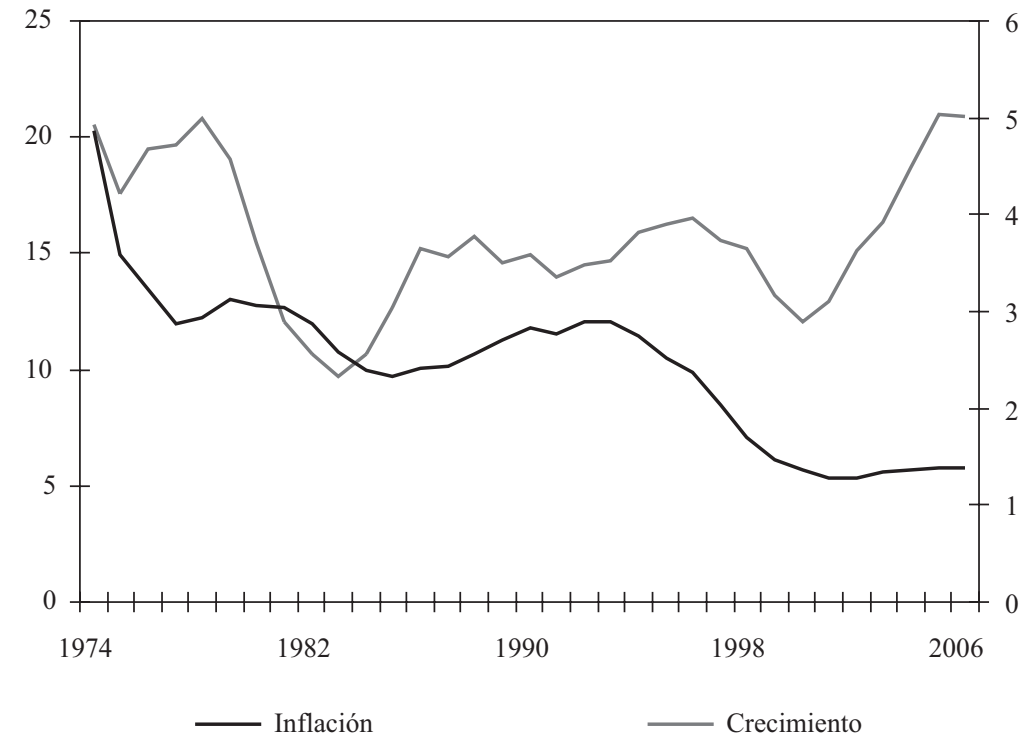

Nota: La inflación se calculó usando la variación porcentual año con año en los precios al consumidor, tomados de la línea 64 del IFS. El crecimiento se calculó como la variación porcentual año con año del volumen de PIB, tomada de la línea 99b del IFS. Los promedios cubren a todos los países que tienen datos disponibles desde 1975. La condición de economía industrializada o en desarrollo se tomó directamente del IFS.

Fuente: FMI: International Financial Statistics (IFS).

dos habrían sido afortunados en los ochenta, y se habrían demorado más de una década en ayudar a los países en desarrollo. Igualmente, el argumento de que la productividad aumentó gracias a — por ejemplo— un mejor manejo de las existencias, tiene problemas parecidos. Cuesta creer que las empresas de los países en desarrollo vinieran a adoptar estas técnicas eficientes más de diez años después ${ }^{3}$.

Sin embargo, las reformas a la política monetaria tardaron largo tiempo antes de ser adoptadas en las economías en desarrollo. La autonomía de

${ }^{3}$ Un argumento similar a favor de la hipótesis de la "buena suerte" plantea Summers (2005). Sostiene que la caída de la volatilidad del crecimiento no fue sincrónica entre todos los países industrializados frente a la reducción común del precio del petróleo. Al mirar las economías en desarrollo el argumento es más sólido, ya que se puede aplicar también al manejo de existencias y otros shocks comunes a la productividad dado que la asincronía es mucho más significativa. 
FIGURA N ${ }^{\circ}$ 2: $\quad$ VOLATILIDAD DE LA INFLACIÓN Y DEL CRECIMIENTO DEL PRODUCTO EN LAS ECONOMÍAS EN DESARROLLO
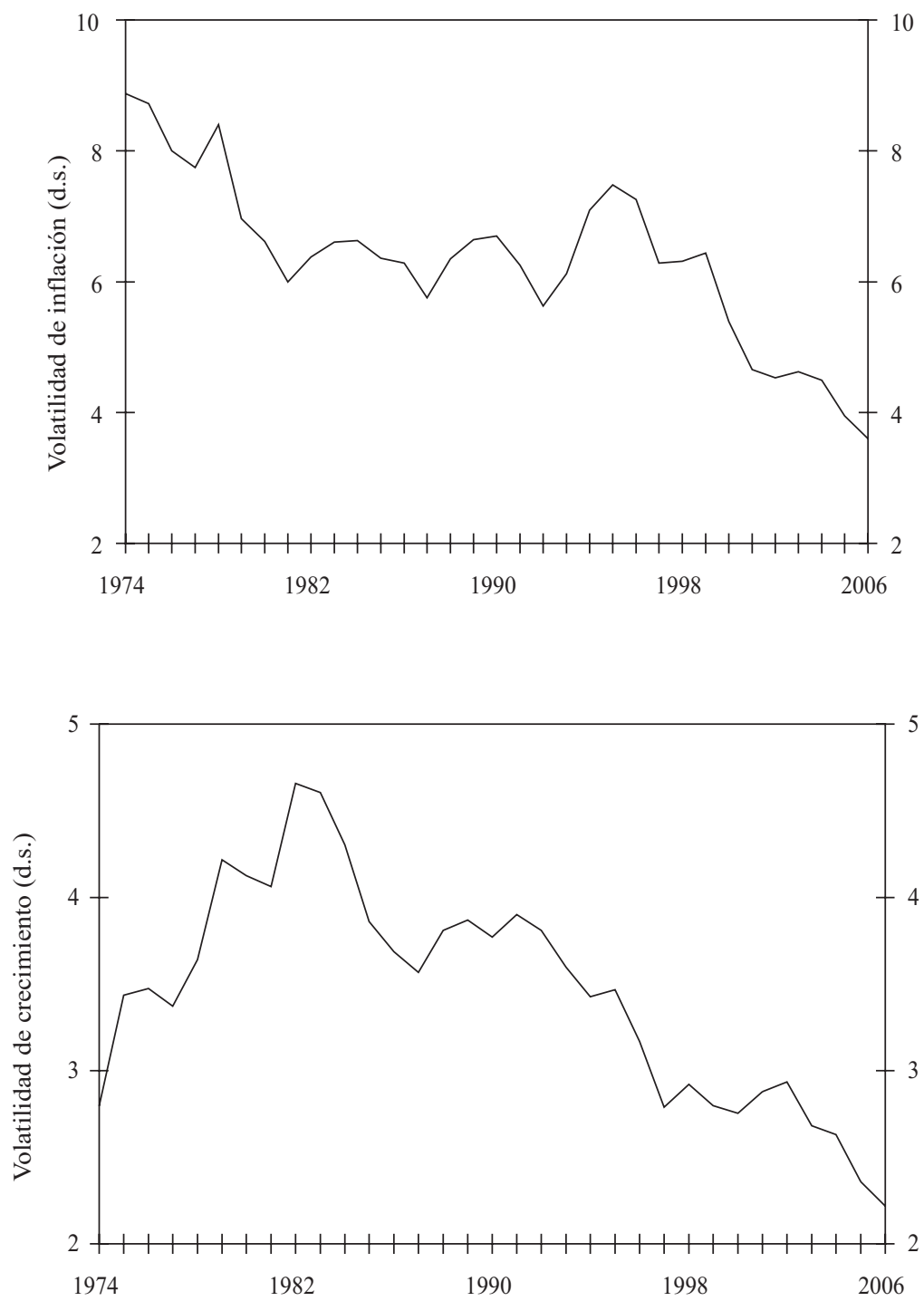

Nota: La inflación y el crecimiento se calcularon del mismo modo que en la Figura $\mathrm{N}^{\circ} 1$ y para los mismos grupos de países. La volatilidad corresponde a la desviación estándar sobre una ventana móvil de cinco años.

Fuente: FMI: International Financial Statistics (IFS). 
los bancos centrales, las metas de inflación y otras reformas sólo llegaron a nuestros países a comienzos de los noventa, coincidiendo con la caída de la volatilidad en las economías en desarrollo. Si bien los cambios estructurales, tales como la apertura internacional, pueden haber favorecido la estabilidad de las economías, dichos cambios también deben incluir los relativos a la política monetaria, porque la Gran Moderación coincide con el control de la inflación, lo que sugiere que la mejora de la política monetaria sin duda jugó un papel importante.

De modo que la pregunta que surge es cómo hace exactamente la política monetaria para reducir la volatilidad del producto y de la inflación. Sabemos que existe un tradeoff entre la volatilidad del producto y la variabilidad de la inflación, que la política monetaria trata de manejar de manera óptima. La autoridad tiene que decidir cómo reducir la inflación para alcanzar la meta. Si reacciona con fuerza ante cualquier desviación de la inflación, el resultado será que la inflación tendrá baja volatilidad, pero la del producto será alta. O sea que, a largo plazo, hay un tradeoff entre la volatilidad de la inflación y la volatilidad del producto, aunque la inflación no tiene ningún efecto sobre el nivel de producto de pleno empleo.

También es posible practicar una política monetaria ineficiente, que esté por debajo de la frontera de volatilidad producto/inflación, aumentando ambas hasta niveles ineficientes. Bernanke (2004) argumenta que en tal escenario de política monetaria ineficiente se puede reducir la volatilidad tanto del producto como de los precios mejorando las políticas. En otras palabras, en presencia de una política monetaria inadecuada podríamos tener volatilidad excesiva del producto y de la inflación, por lo que mejorar el manejo monetario puede permitirnos alcanzar una frontera eficiente, lo que reduciría ambas volatilidades.

Por ejemplo, una política monetaria que no reacciona con suficiente energía a un cambio en la inflación, que no cumple con el principio de Taylor, o una política demasiado optimista respecto del nivel de producto de pleno empleo, podría acabar generando una volatilidad de producto e inflación ineficientemente altas. Éstas son explicaciones razonables para la forma en que se condujo la política monetaria en Estados Unidos antes de la deflación de los ochenta. Más aún, estas ineficiencias de política podrían agravarse si se cree que existe un tradeoff permanente entre desempleo e inflación, lo que podría llevar a la hipótesis aceleracionista de Friedman.

Quisiera, sin embargo, subrayar las ventajas de la credibilidad que trae consigo el compromiso con la inflación estable. En una curva de Phillips estándar, la inflación depende de la brecha de actividad (el motivo por el cual existe el dilema entre volatilidad del producto y de la inflación), 
de las expectativas inflacionarias y de un conjunto de otras variables que incluyen los shocks inflacionarios. Analicemos el caso de un objetivo inflacionario bajo con poca credibilidad. Ahí, un shock inflacionario puede retroalimentar la formación de precios y salarios, y requerir una respuesta más agresiva de la política monetaria, aumentando el costo de reducir la inflación. Esto puede suceder cuando las expectativas tienen un alto grado de inercia debido a un alto nivel de indexación o poca credibilidad. Por contraste, cuando las expectativas están ancladas en la meta de inflación, se requiere un ajuste menor de la política monetaria para lograr una inflación baja y estable, y el sacrificio es menor, de manera que cae la volatilidad tanto de la inflación como del producto ${ }^{4}$.

Obviamente, no es fácil lograr la credibilidad, pero los esfuerzos pueden ir por varios caminos. Sin duda la conquista de una inflación baja, como ha ocurrido en los países en desarrollo, aumenta la confianza en la competencia de las autoridades y su compromiso con el control de la inflación. Ello deriva en expectativas de inflación ancladas y reduce la persistencia inflacionaria. Una meta de inflación, con el apoyo institucional necesario como es un banco central autónomo con un mandato claro de estabilidad de precios, contribuye a su logro. Establece el compromiso explícito de mantener la inflación baja y estable en el tiempo, y una estrategia para ajustarse a las desviaciones, que incluye el horizonte temporal en el que debe lograrlo. De hecho, entre las economías en desarrollo, las que han adoptado el esquema de metas de inflación han podido ostentar menor volatilidad inflacionaria y del producto que las demás (Figura $N^{\circ} 3$ ). Por supuesto, esta evidencia no es concluyente y la causalidad podría ir en sentido opuesto, o sea que las economías más estables serían más propensas a implementar un esquema de metas de inflación. La evidencia empírica es mixta, pero en general se ha visto que las metas de inflación sí reducen tanto el nivel como la volatilidad de la inflación ${ }^{5}$. Existe alguna evidencia para las economías emergentes de que las metas de inflación también reducen la volatilidad del crecimiento (Gonçalves y Salles, 2008).

\footnotetext{
${ }^{4}$ Para un modelo sobre este punto, véase De Gregorio (2007).

${ }^{5}$ Truman (2003), Hyvonen (2004) y Vega y Winkelried (2005), entre otros. Ball y Sheridan (2005) muestran que la evidencia disponible no apoya esta conclusión para las economías desarrolladas. Mishkin y Schmidt-Hebbel (2007) corroboran esta diferencia entre economías desarrolladas y emergentes, y concluyen que quienes ganan son las economías emergentes y los países con metas de inflación que convergen a la meta. Sin embargo, concluyen también que la elección del grupo de control es clave para encontrar algún efecto de las metas de inflación sobre la inflación efectiva.
} 
FIGURA N ${ }^{\circ}$ 3: $\quad$ VOLATILIDAD DE LA INFLACIÓN EN LAS ECONOMÍAS EN DESARROLLO: PAÍSES CON Y SIN METAS DE INFLACIÓN

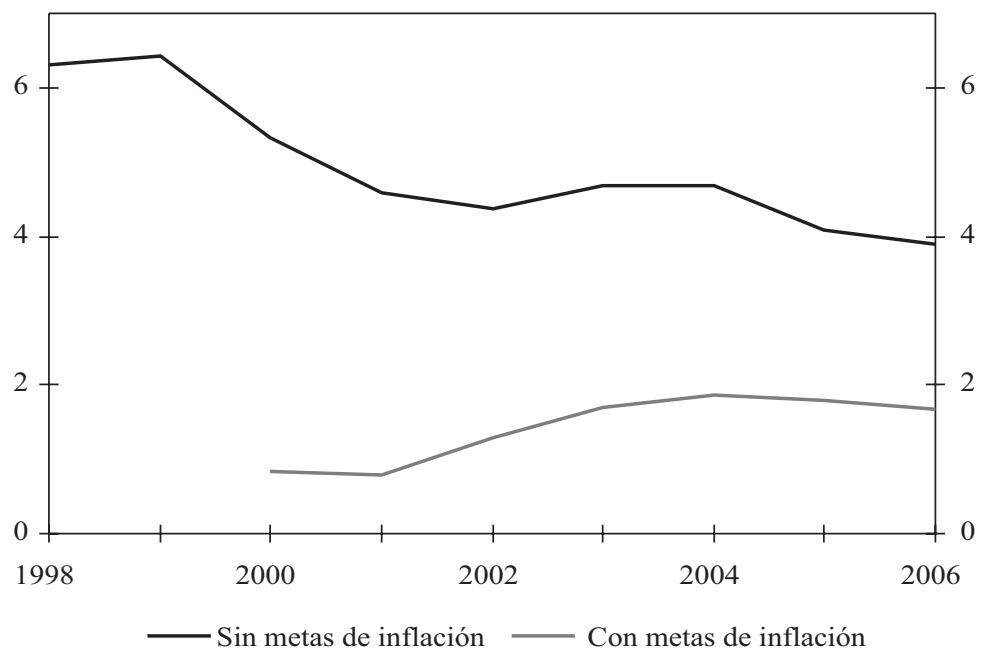

Nota: La volatilidad de la inflación se calcula del mismo modo que en la Figura $\mathrm{N}^{0} 1$ y para el mismo grupo de países, separados entre aquellos con y sin metas de inflación. Se considera con metas de inflación aquellos países que durante la ventana de tiempo correspondiente permanecen más del $50 \%$ del tiempo en su régimen.

Fuente: FMI: International Financial Statistics (IFS).

\section{La mala suerte está de vuelta: Los riesgos de la inflación}

En el último tiempo, el mundo ha vivido uno de los peores shocks inflacionarios en sesenta años. Primero fue el petróleo, que comenzó a subir casi verticalmente a comienzos de la década, y ha continuado superando sus propios récords (Figura $\mathrm{N}^{\circ} 4$ ). Sin embargo, a pesar de este shock, tanto la inflación como el producto han mostrado una volatilidad mucho más baja que en los ciclos anteriores del precio del petróleo. Una menor dependencia petrolera y políticas fiscales contracíclicas explican en parte estos efectos menores. También mejores políticas monetarias han aportado en forma sustancial a aminorar el impacto de los shocks petroleros sobre el producto y la actividad $^{6}$. Sin embargo, el petróleo ha seguido subiendo, y aunque sus efectos son hoy menores que antes, el shock está creciendo tanto y con

${ }^{6}$ De Gregorio et al. (2007); Blanchard y Galí (2007) y Galí y Gambetti (2007) muestran evidencia que apoya estas ideas. 
FIGURA N ${ }^{\circ}$ 4: $\quad$ PRECIO REAL DEL PETRÓLEO WTI

(dólares de febrero 2008 el barril)

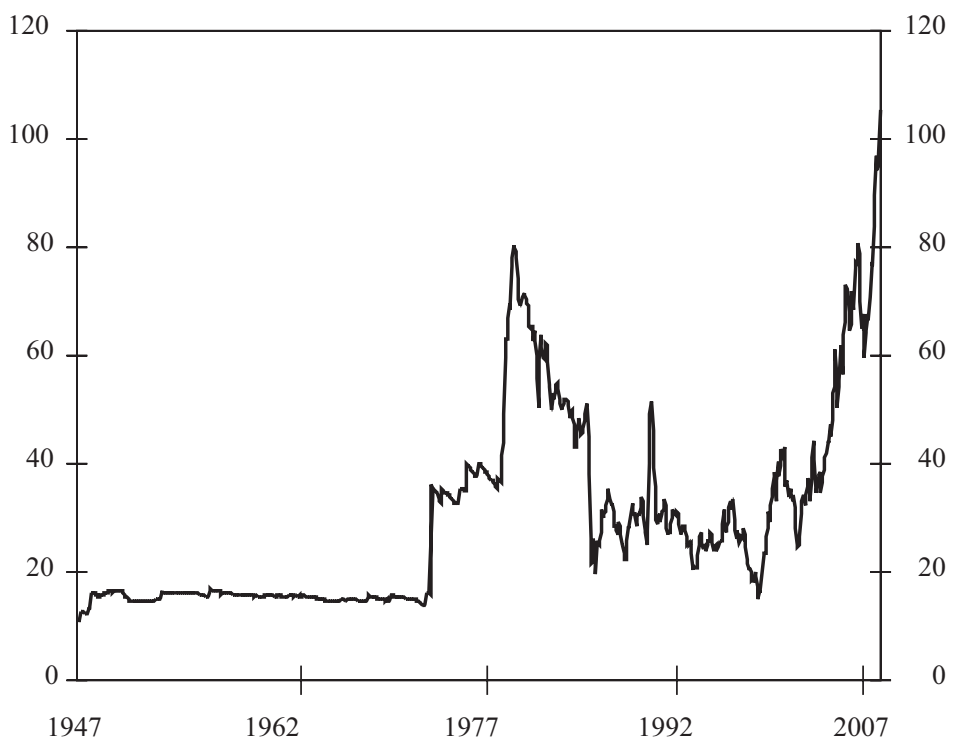

Nota: Precio deflactado por el índice de precios al productor de EE.UU.

Fuente: Bloomberg.

tanta persistencia que en los próximos trimestres puede tener efectos importantes sobre la inflación y el producto. El lado bueno es que los mejores esquemas de política monetaria que se utilizan hoy permiten atenuar estos efectos.

Por otro lado, últimamente hemos visto un aumento impresionante de los precios de los alimentos (Figura $\mathrm{N}^{\circ} 5$ ), con graves efectos inflacionarios en varios países. En algunos casos los alimentos han llegado a máximos históricos, cuyas causas principales son la mayor demanda de algunas economías emergentes como China, India y algunos países exportadores de petróleo, el uso de granos para producir biocombustibles, y algunos factores financieros asociados a los inversionistas y sus posiciones largas en recursos naturales. Desde mediados del 2006, los precios de los granos medidos por el índice CRB, han crecido a tasas anuales de 56\%, cuando antes, desde el año 1990, el crecimiento anual era de un medio por ciento. Esta tendencia es común para otras materias primas. El trigo venía bajando alrededor de 1\% desde 1990 hasta mitad del 2006, y desde entonces ha subido $121 \%$, contando una reversión parcial el último mes. También el 
FIGURA N ${ }^{\circ}$ 5: $\quad$ PRECIOS DE ALIMENTOS

(Índice, enero $2006=100$ )

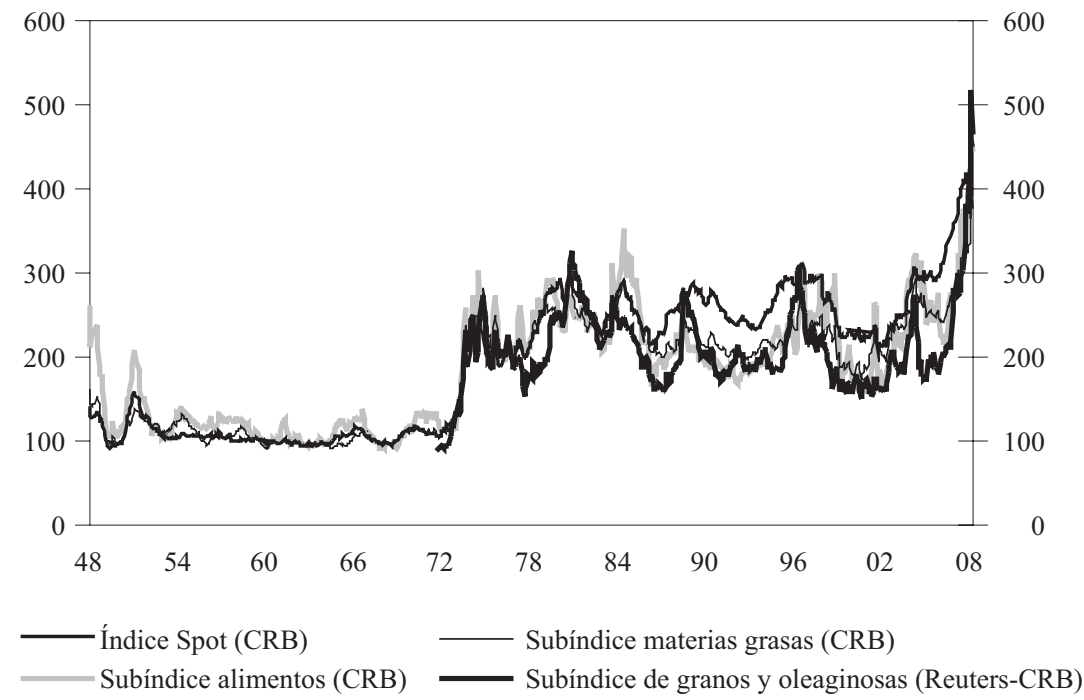

Fuentes: Commodity Research Bureau, Reuters y Bloomberg.

arroz, cuyo precio prácticamente no cambió en el primer período estudiado, ha venido escalando a 71\% anual desde mediados del 2006. La leche, por último, tras bajar de precio a una tasa anual del 0,3\% entre 1990 y mediados del 2006, pasó a 70\% anual de crecimiento promedio.

Los efectos directos del shock se han sentido en alzas abruptas de alimentos y energía, mientras los demás precios han mostrado un buen comportamiento (Figura $\mathrm{N}^{\circ}$ 6). Por ejemplo, en Chile la inflación fluctúa alrededor de $8 \%$, cuatro puntos más arriba del techo del rango meta (2 a 4\%), principalmente por culpa de los alimentos y la energía. De hecho, la inflación de los otros precios sigue cerca del 3\%. Cabe señalar que los alimentos han subido además por problemas climáticos, como las heladas del 2007 y la sequía de este año.

La naturaleza de estas alzas es puramente internacional o climática, lo que para la autoridad monetaria es exógeno. Sin embargo, su posible propagación a otros precios y a los salarios a través de lo que se conoce como efectos de segunda vuelta asigna un rol importante a la política monetaria aun frente a estos shocks específicos a ciertos precios externos. Dada la naturaleza del shock, que se debe en gran parte a la producción de bio- 
FIGURA N ${ }^{\circ}$ 6: $\quad$ INFLACIÓN: ALIMENTOS, ENERGÍA Y RESTO

(Variación anual, porcentaje)

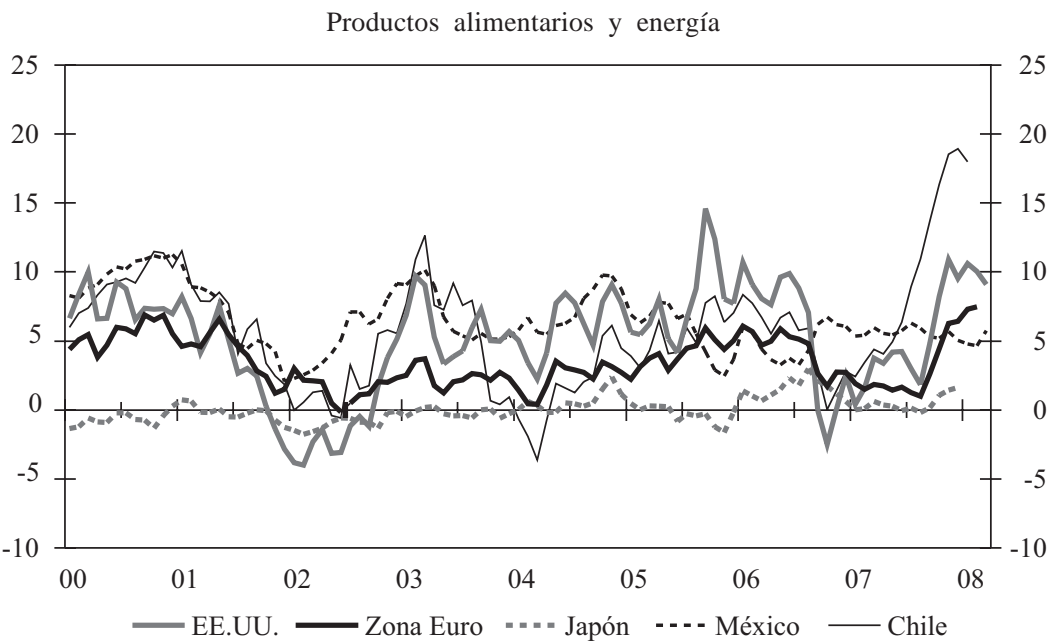

Productos no alimentarios y energía

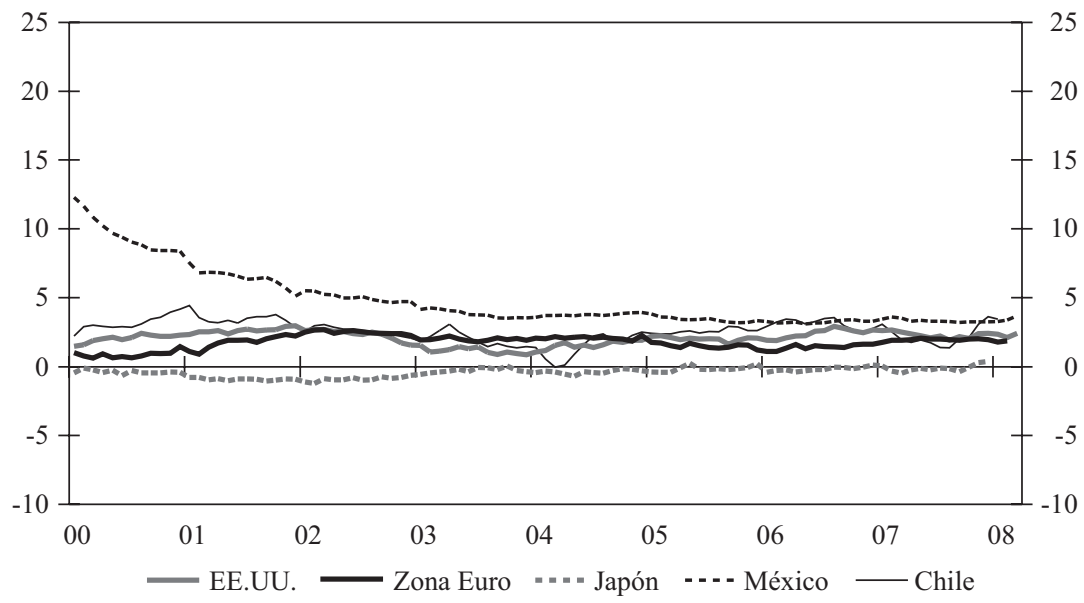

Fuentes: Bancos centrales y Bloomberg.

combustibles y a la creciente demanda de alimentos de estos nuevos actores asiáticos, lo probable es que el shock persista por un buen tiempo. Algunos efectos de segunda vuelta son inevitables, por el grado de inercia que siempre tiene la formación de salarios y precios. El rol de la política monetaria es asegurar que los precios relativos se ajusten sin provocar un 
aumento persistente en la inflación, esto es, más allá de su dinámica normal. Dicho de otro modo, la política monetaria debe garantizar que la dinámica de los precios sea coherente con que la inflación converja a su meta, dentro del horizonte de política. Así, la política monetaria se debe preocupar - y ocupar- de la dinámica de inestabilidad que puede generar aumentos de la inflación que luego sean demasiado costosos de contener. Estos son los efectos indeseados de segunda vuelta que pueden requerir de drásticas medidas de política monetaria.

En un esquema de metas de inflación, cuando ésta se desvía de la meta, se utiliza la política monetaria para hacer regresar la inflación proyectada a su nivel meta dentro de un horizonte de tiempo específico, u horizonte de política. En Chile, esto se traduce en hacer que la inflación proyectada sea de $3 \%$ en un horizonte de dos años. La extensión de este horizonte se define en función de los rezagos con que se materializan los efectos de la política monetaria, y teniendo en cuenta que manejar la inflación tiene un costo en producto. También permite que los shocks transitorios reviertan sus efectos sin alterar la trayectoria de la política monetaria. Por lo tanto, en el contexto actual de fuertes presiones inflacionarias, la política monetaria debe evitar los efectos indeseados de segunda vuelta, que son aquellos que generan un ajuste más largo que el que cubre el horizonte de política.

A fin de monitorear estos efectos de segunda vuelta potencialmente perjudiciales, escudriñamos las dinámicas de precios que parecen haberse salido del camino. Por ejemplo, seguimos la evolución de los salarios y la contrastamos con las cláusulas de reajuste o indexación históricas. Si están subiendo más de lo prudente, podríamos estar en presencia de una dinámica salarial que puede hacer necesaria una reacción de política.

En este escenario, se podría argumentar a favor de relajar el horizonte o el nivel de la meta para acomodar los shocks inflacionarios sin que el producto se resintiera. Pero, como ya he comentado, esto podría ser muy perjudicial a futuro. Renunciar al control de la inflación nos haría perder el ancla inflacionaria de las expectativas, resultando en una fijación de precios y salarios más retrospectiva que prospectiva, y un aumento de la volatilidad del producto. En otras palabras, obtendríamos un resultado ineficiente para la política monetaria y perderíamos los beneficios de la Gran Moderación.

En consecuencia, los bancos centrales deben ejercer un cuidadoso monitoreo de las expectativas inflacionarias. Sin embargo, en la actual coyuntura de incertidumbre extrema y alta volatilidad inflacionaria, las medidas de compensación, que comparan los activos financieros nominales y reajustables que típicamente seguimos, han aumentado significativamente en todo el mundo, incluido Chile, lo que parece indicar un importante aumento 
de las expectativas, posiblemente reflejando que se ha perdido el ancla nominal. Este fenómeno de aumentos, que refleja un premio por riesgo en las proyecciones de inflación en el mundo, se ve agudizado en Chile por la profundidad del mercado de instrumentos indexados a la inflación que los convierte en un refugio natural contra la inflación. Por lo tanto, en tiempos de incertidumbre como los que estamos atravesando, las medidas de compensación inflacionaria pueden aumentar sin que necesariamente impliquen un desanclaje de las expectativas, haciendo más complicado reconocerlo en forma oportuna.

Se podría argumentar — erradamente, a mi juicio— que una forma de evitar la reacción de la política monetaria ante un shock inflacionario específico sería enfocarse en controlar la inflación subyacente en lugar de la inflación del IPC. Este tema levanta mucha polémica, pero en el contexto de esta discusión quisiera agregar que más allá del problema de modificar la meta en forma oportunista y el impacto que tendría en la credibilidad, hay una razón más profunda por la que fijar un objetivo para la inflación subyacente en lugar del IPC no es opción en el contexto actual, en que los precios de las materias primas ya subieron muchísimo. En el presente escenario, los efectos de segunda vuelta se manifiestan en todos los precios. Por ejemplo, un aumento fuerte de los salarios originado en la indexación a la inflación pasada, o en otro tipo de inercia, afecta a todos los precios por la vía de los costos y, en consecuencia, la dinámica perniciosa de la inflación aparecerá tanto en la inflación del IPC como en la inflación subyacente. En realidad, un ajuste razonable debería hacer que la inflación de los alimentos y de la energía cayera en forma drástica luego que se hubiera producido el ajuste de precios relativos, y que la inflación subyacente se ajustara desde un nivel más alto hacia la meta. Una vez decantados los precios de alimentos y energía, la propagación de la inflación sería un problema más serio para los países cuya meta fuera la inflación subyacente.

\section{Desafíos de política monetaria}

Los actuales shocks inflacionarios son una seria amenaza para la política monetaria. El ajuste reciente ha sido dispar entre países. Muchos han tratado de evitar las alzas mediante subsidios, impuestos y/o directamente controlando los precios. Sin embargo, a estas alturas sabemos que los precios relativos tienen que ajustarse, dada la persistencia del shock y la necesidad de contar con señales apropiadas para asegurar la asignación eficiente de los recursos. Por otro lado, en algunos países - Chile inclui- 
do- las variaciones de los costos son normalmente traspasados a los precios finales, porque los mercados de alimentos están globalmente integrados y son competitivos. En consecuencia, gran parte del shock inflacionario ya fue absorbido. Aun así hemos sido testigos de un flujo continuo de malas noticias en los precios de los recursos naturales, y seguimos siendo golpeados por shocks inflacionarios, pero debemos conservar las ventajas que trae el esquema flexible de metas de inflación en términos de estabilidad de precios y credibilidad. No hay duda, desde la perspectiva de país en desarrollo, que lo anterior es una conquista importante que no podemos abandonar, y menos que nunca en momentos difíciles.

La economía mundial está viviendo una situación muy compleja. No es tan desastrosa como fueron los setenta, pero estamos en presencia de una desaceleración con un alto riesgo de inflación. La política monetaria está siendo puesta a prueba con un test de tensión que será crucial para identificar futuros refinamientos de los esquemas de política. De particular importancia en Estados Unidos y en las economías industrializadas es la interacción entre la estabilidad de precios y la estabilidad financiera. Desde el punto de vista del manejo de las políticas macroeconómicas, debemos asegurar que todos los beneficios logrados durante la fase de la Gran Moderación se conserven. Sería una irresponsabilidad retornar a los tiempos de inestabilidad por buscar ganancias que duran poco.

\section{REFERENCIAS}

Ahmed, S., A. Levin y B. Wilson (2002): "Recent U.S. Macroeconomic Stability: Good Policies, Good Practices, or Good Luck?”. Board of Governors of the Federal Reserve System, International Financial Discussion Paper 2002-730.

Ball, L. y N. Sheridan (2005): “Does Inflation Targeting Matter?”. En B. Bernanke y M. Woodford (eds.), The Inflation-Targeting Debate. University of Chicago Press.

Blanchard, O. y J. Galí (2007): "The Macroeconomic Effects of Oil Price Shocks: Why Are the 2000s So Different from the 1970s?”. Working Paper Series $\mathrm{N}^{\circ} 13368$, NBER.

Blanchard, O. y J. Simon (2001): “The Long and Large Decline in US Output Volatility”. En Brookings Papers on Economic Activity, (1) pp. 135-164.

Bernanke, B. (2004): “The Great Moderation”. Comentarios del presidente de la Reserva Federal en el encuentro de Eastern Economic Association, Washington, DC.

Betancour, C., J. De Gregorio y J. Medina (2008): "The Great Moderation and the Monetary Transmission Mechanism in Chile”. En BIS Papers $N^{\circ} 35$, Bank of International Settlements, pp. 159-178.

Cavallo, E. (2007): “Output Volatility and Openness to Trade: A Reassessment”. Working Paper $N^{\circ}$ 604, Inter-American Development Bank.

Cecchetti, S., A. Flores-Lagunes y S. Krause (2005): "Sources of Changes in the Volatility 
of Real Growth”. Presentado en seminario organizado por el RBA. En C. Kent y D. Norman (eds.), The Changing Nature of the Business Cycle. Reserve Bank of Australia (RBA).

De Gregorio, J. (2007): "Defining Inflation Targets, the Policy Horizon and the OutputInflation Tradeoff”. Working Paper $\mathrm{N}^{\circ} 415$, Banco Central de Chile.

De Gregorio, J., O. Landerretche y C. Neilson (2007): “Another Pass-Through Bites the Dust? Oil Prices and Inflation”. En Economía, 7 (2), (primavera) pp. 155-196.

Galí, J. y L. Gambetti (2007): “On the Sources of the Great Moderation”. Economics Working Papers $\mathrm{N}^{\circ}$ 1041, Universitat Pompeu Fabra.

Gerlach, S., A. Giovannini, C. Tile y J. Viñals (2008): Low Inflation: Testing Times for Central Banks. Geneva Report on the World Economy, 10.

Gonçalves, C. y J. Salles (2008): "Inflation Targeting in Emerging Economies: What Do the Data Say?”. En Journal of Development Economics, 85, pp. 312-318.

Hyvonen, M. (2004): "Inflation Convergence across Countries". Discussion Paper N ${ }^{\circ}$ 2004-04, Reserve Bank of Australia.

Kent, C., K. Smith y J. Holloway (2005): "Declining Output Volatility: What Role for Structural Change?”. Presentado en seminario organizado por el RBA. En C. Kent y D. Norman (eds.), The Changing Nature of the Business Cycle. Reserve Bank of Australia (RBA).

Kim, C.-J. y C. Nelson (1999): "Has the U.S. Economy Become More Stable? A Bayesian Approach Based on a Markov Switching Model of the Business Cycle”. En The Review of Economics and Statistics, 81 (4) pp. 608-616.

McConnell, M. y G. Pérez-Quiroz (2000): "Output Fluctuations in the United States: What Has Changed since the 1980s?”. En American Economic Review, 90 (5), pp. 1464-1476.

Mishkin, F. y K. Schmidt-Hebbel (2007): “Does Inflation Targeting Make a Difference?”. En F. Mishkin y K. Schmidt-Hebbel (eds.), Monetary Policy under Inflation Targeting. Banco Central de Chile, pp. 291-372.

Stock, J. y M. Watson (2003): "Has the Business Cycle Changed? Evidence and Explanations”. En Monetary Policy and Uncertainty: Adapting to a Changing Economy, pp. 9-56, Federal Reserve Bank of Kansas City.

Summers, P. (2005): "What Caused The Great Moderation? Some Cross-Country Evidence”. En Economic Review, Federal Reserve Bank of Kansas City, tercer trimestre, pp. 5-32.

Truman, E. (2003): Inflation Targeting in the World Economy. Washington D.C.: Peter G. Peterson Institute for International Economics.

Vega, M. y D. Winkelried (2005): "Inflation Targeting and Inflation Behavior: A Successful Story?”. En International Journal of Central Banking, 1 (3) pp. 15375. 